\title{
Revised WMAP constraints on neutrino masses and other extensions of the minimal $\Lambda \mathrm{CDM}$ model
}

\author{
Jostein R. Kristiansen, ${ }^{1, *}$ Hans Kristian Eriksen, ${ }^{1,2,3,4, \dagger}$ and Øystein Elgarøy ${ }^{1, *}$ \\ ${ }^{1}$ Institute of Theoretical Astrophysics, University of Oslo, Box 1029, 0315 Oslo, Norway \\ ${ }^{2}$ Centre of Mathematics for Applications, University of Oslo, Box 1053 Blindern, 0316 Oslo, Norway \\ ${ }^{3}$ Jet Propulsion Laboratory, 4800 Oak Grove Drive, Pasadena California 91109, USA \\ ${ }^{4}$ California Institute of Technology, Pasadena, California 91125, USA
}

(Received 1 August 2006; published 13 December 2006)

Recently, two issues concerning the three-year Wilkinson Microwave Anisotropy Probe (WMAP) likelihood code were pointed out. On large angular scales $(l \lesssim 30)$, a suboptimal likelihood approximation resulted in a small power excess. On small angular scales $(l \gtrsim 300)$, over-subtraction of unresolved point sources produced a small power deficit. For a minimal six-parameter cosmological model, these two effects conspired to decrease the value of $n_{s}$ by $\sim 0.7 \sigma$. In this paper, we study the change in preferred parameter ranges for extended cosmological models, including running of $n_{s}$, massive neutrinos, curvature, and the equation of state for dark energy. We also include large-scale structure and supernova data in our analysis. We find that the parameter ranges for $\alpha_{s}, \Omega_{k}$ and $w$ are not much altered by the modified analysis. For massive neutrinos the upper limit on the sum of the neutrino masses decreases from $M_{\nu}<1.90 \mathrm{eV}$ to $M_{\nu}<1.57 \mathrm{eV}$ when using the modified WMAP code and WMAP data only. We also find that the shift of $n_{s}$ to higher values is quite robust to these extensions of the minimal cosmological model.

DOI: 10.1103/PhysRevD.74.123005

PACS numbers: $98.70 . V c, 95.35 .+d, 98.80 . C q$

\section{INTRODUCTION}

The temperature fluctuations in the cosmic microwave background $(\mathrm{CMB})$ radiation have proved to be the single most important cosmological observable we have today, and the high-precision full-sky maps provided by the Wilkinson Microwave Anisotropy Probe (WMAP) play a very important role in the determination of cosmological parameters and preferred cosmological models [1]. One of the most important conclusions both from the WMAP data alone, and from joint analyses including other cosmological observables, is that a simple six-parameter flat $\Lambda \mathrm{CDM}$ model fits the data very well, and that extended models with additional free parameters do not improve the fit significantly.

Because of the great impact of WMAP data, the 3-year data analysis from the WMAP team has been subject to exhaustive cross-checking. In particular, in Refs. [2,3] two noticeable issues with the likelihood code as first presented by the WMAP team were pointed out. First, the likelihood approximation used between $13 \leq l \leqq 30$ appears to be inadequate, effectively resulting in a $\sim 5 \%$ power excess in this range compared to an exact treatment. Second, the amplitude for the unresolved point source spectrum used by the WMAP team was found to over-subtract the actual contribution in the data, leading to a power deficit at high l's.

\footnotetext{
*Electronic address: j.r.kristiansen@astro.uio.no

${ }^{\dagger}$ Electronic address: h.k.k.eriksen@astro.uio.no

Electronic address: oelgaroy@astro.uio.no
}

In [3], the effect of these discrepancies were studied for a minimal six-parameter cosmological model. This was done both for WMAP data only, and with additional CMB data from small-scale experiments. Their main finding was an increase in $n_{s}$, lowering the significance of $n_{s} \neq$ 1 from $\sim 2.7 \sigma$ to $\sim 2.0 \sigma$.

In this paper we consider the effect on extended cosmological models. We study how the modified WMAP likelihood affects the preferred ranges of the running of the scalar spectral index, the cosmological neutrino mass limits, spatial curvature, and the equation of state for dark energy. We have also taken into account large-scale structure (LSS) and type 1a supernovae (SNIa) data sets, to see whether the shifts in preferred parameter ranges survive a more thorough cosmological analysis. Further, we consider whether the shift of $n_{s}$ to larger values is robust to changes in cosmological models and data sets.

In the next section, we review both the methods and data we use. In Sec. III, we report and comment upon our results, before summarizing and concluding in Sec. IV.

\section{DATA AND METHODS}

\section{A. WMAP data}

The WMAP experiment is a NASA-funded satellite mission designed to measure the CMB temperature anisotropies over the full sky at five frequencies between 23 and $94 \mathrm{GHz}$ with unprecedented angular resolution and sensitivity. These measurements allow for an accurate determination of the angular CMB power spectrum for angular scales between, say, $l=2$ and 800 with three years of observations. 
Estimation of this spectrum and the corresponding likelihood function is a multistep process. First, sky maps are generated from the raw satellite data, and the instrumental noise is estimated. Second, contaminants in the form of galactic and extragalactic foregrounds are removed from the sky maps, and severely contaminated regions are removed completely from further analysis. Third, the power spectrum is estimated with some algorithm, usually trading off computational efficiency against accuracy. Fourth, a connection is made between the power spectrum and the likelihood.

These steps are all described in detail for the three-year WMAP data release in Ref. [4]. The main result of these efforts is a user-friendly Fortran 90 code that for an input power spectrum outputs the corresponding likelihood value. In principle, this piece of code may be used as a "black box."

However, some care is warranted. In particular, two points were noted in Ref. [2]. First, there is a $\sim 5 \%$ discrepancy between the temperature likelihood approximation used by the WMAP team and an exact evaluation for $l \lesssim 30$. Second, there is a $\sim 60 \mu \mathrm{K}^{2}$ discrepancy between the two spectra observed at 61 and $94 \mathrm{GHz}$. The former is primarily due to estimator approximations and secondarily to residual foregrounds. The latter issue was later partly explained in terms of an excessive point source correction applied to the WMAP spectrum [3].

In the present paper, we therefore use two versions of the WMAP likelihood. The first version is simply the official code as provided on LAMBDA [5]. The second version includes two modifications to this code: At $l \leq 30$, we replace both the WMAP pixel-based likelihood and the pseudo- $C_{l}$-based likelihood with an exact Gibbs sampling based estimator [2]. Then the spectrum amplitude of unresolved point sources (relative to $41 \mathrm{GHz}$ ) is adjusted from $A=0.017 \mu \mathrm{K}^{2}$ sr to $A=0.011 \mu \mathrm{K}^{2}$ sr [3]. We do not marginalize over the SZ (Sunyaev-Zeldovich) amplitude in our analyses.

\section{B. Other data sets used}

In our analysis we use additional CMB data, data from LSS surveys, SNIa data and additional priors on the Hubble parameter and baryon content of the universe.

\section{Other CMB observations}

To probe a larger range of angular scales in the CMB power spectrum we use CMB data from ACBAR [6] and BOOMERanG [7-9].

\section{Large scale structure}

Large scale structure surveys probe the matter distribution in the universe by measuring the galaxy-galaxy power spectrum $P_{g}(k, z)=\left\langle\left|\delta_{g}(k, z)\right|^{2}\right\rangle$. In the linear perturbation regime it is expected that this galaxy-galaxy spectrum is proportional to the total matter power spectrum, $P_{m}$, through the simple relation $P_{g}=b^{2} P_{m}$, where $b$ is called the bias parameter.

There are two galaxy surveys of comparable size, namely, the 2 degree Field Galaxy Redshift Survey (2dF) [10] and the Sloan Digital Sky Survey (SDSS) [11]. In our analysis we use data from both these surveys.

\section{Type 1a supernovae}

Probing the luminosity-redshift relation of SNIa is one of the most direct measurements of cosmological expansion, and thus one of the most powerful pieces of evidence for the existence of dark energy. In our analysis we use SN1a data from the Supernova Legacy Survey (SNLS) [12], which is a dedicated SNIa survey currently including 71 SNIa in the redshift range $z=0.2-1$.

\section{Additional priors}

In addition to the CMB, LSS and SNIa data sets mentioned above, we impose priors on the Hubble parameter, the baryon content in the universe, and the position of the LSS baryonic peak.

From the Hubble Space Telescope Key Project (HST) we have adopted a prior on the Hubble parameter of $h=$ $0.72 \pm 0.08$ [13]. The constraint on the baryon density today was chosen to be $\Omega_{b} h^{2}=0.022 \pm 0.002$ from big bang nucleosynthesis (BBN) $[14,15]$.

From the detection of baryonic acoustic oscillations (BAO) in the sample of luminous red galaxies (LRG) in the SDSS survey [16] it is also possible to put a constraint on the combination of parameters

$$
A_{\mathrm{BAO}} \equiv\left[D_{M}(z)^{2} \frac{z}{H(z)}\right]^{1 / 3} \frac{\sqrt{\Omega_{m} H_{0}^{2}}}{z},
$$

where $D_{M}(z)$ is the comoving angular diameter distance. The BAO constraint can then be written as

$$
A_{\mathrm{BAO}}=0.469\left(\frac{n_{s}}{0.98}\right)^{-0.35}\left(1+0.94 f_{\nu}\right) \pm 0.017,
$$

where the fit to the neutrino fraction, $f_{\nu}=\Omega_{\nu} / \Omega_{m}$ is given by [17]. For $z$ we adopt the redshift of a typical LRG in the SDSS sample, $z=0.35$. For models with nonzero $\alpha_{s}$, we substitute $n_{s}$ in Eq. (2) with an effective $n_{s}$ given by

$$
\left.n_{s, \mathrm{eff}}\left(k_{1}\right) \equiv \frac{d \ln P}{d \ln k}\right|_{k=k_{1}}+1=n_{s}\left(k_{0}\right)+\alpha_{s} \ln \left(k_{1} / k_{0}\right),
$$

where $P$ is the primordial power spectrum given by

$$
\ln P=\ln A_{s}+\left(n_{s}-1\right) \ln \left(k / k_{0}\right)+\alpha_{s} / 2 \ln \left(k / k_{0}\right)^{2},
$$

and $k_{0}$ is set to $0.05 \mathrm{Mpc}^{-1}$. We use $k_{1}=0.01 \mathrm{~h} \mathrm{Mpc}^{-1}$, which is approximately the scale associated with the bar- 
TABLE I. The different combinations of data sets used in this analysis.

\begin{tabular}{ll}
\hline \hline \multicolumn{2}{l}{ Data set Observations included } \\
\hline A & WMAP \\
B & WMAP + ACBAR + BOOMERanG \\
C & WMAP + ACBAR + BOOMERanG $+\mathrm{SDSS}+2 \mathrm{dF}+$ \\
& SNLS + HST + BBN + BAO \\
\hline \hline
\end{tabular}

yonic peak in the LRG sample. Our results are robust to changes of $k_{1}$ around this value, as the preferred values of $\alpha_{s}$ are small in the models considered here.

\section{Parameter estimation}

The parameter estimation process is based on the publicly available CosmoMC code [18], using the data and likelihoods described above.

For each model, we compute the corresponding parameter confidence intervals using three different combinations of data sets, named A, B and C (see Table I). Data set A includes the three-year WMAP data only; data set B includes also CMB data from ACBAR and BOOMERanG; and data set $\mathrm{C}$ also LSS and SNIa data sets and priors from HST, BBN and BAO.

As a basic six-parameter cosmological model we use the parameters $\left\{\Omega_{b} h^{2}, \Omega_{m}, \log \left(10^{10} A_{s}\right), h, n_{s}, \tau\right\}$. The exact parameter definitions are given by the CosmoMC code. $\Omega_{b}$ is the ratio of baryons to the total energy density; $h$ is the Hubble parameter today; $\Omega_{m}$ is the ratio of matter to the total energy density today; $A_{s}$ sets the amplitude of the primordial fluctuations; $n_{s}$ is the tilt of the primordial power spectrum; and $\tau$ is the reionization optical depth.

We then extend the six-parameter model by adding the parameters $\alpha_{s}, r, M_{\nu}, \Omega_{k}, w$ one by one (except for $\alpha_{s}$ and $r$ which are added simultaneously). Here, $\alpha_{s}$ is the running of $n_{s}$, defined in Eq. (4); $r$ is the ratio of tensor to scalar fluctuations; $M_{\nu}$ is the sum of the neutrino mass eigenstates, $M_{\nu}=\sum m_{\nu}=93.14 \Omega_{\nu} h^{2} ; \Omega_{k}$ is the amount of spatial curvature; and $w$ is the equation of state parameter for dark energy. Finally, we vary all 11 parameters simultaneously.

For all combinations of data and parameter sets, we carry out a similar analysis both with the standard WMAP likelihood code as provided and with the two modifications described above.

\section{RESULTS}

\section{A. Minimal six-parameter model}

We start with the simple six-parameter model having the free parameters $\left\{\Omega_{b} h^{2}, \Omega_{m}, \log \left(10^{10} A_{s}\right), h, n_{s}, \tau\right\}$. This was first done in Ref. [3] for the combinations of data sets A and $\mathrm{B}$ (see Table I). For $n_{s}$ they reported a $\sim 0.7 \sigma$ shift to higher values when applying the modified analysis. The
TABLE II. Results for $n_{s}$ in a six-parameter model. The values in the second column are found using the WMAP likelihood code, while the values in the third column are calculated using the modifications described in section II A. All errors are $1 \sigma$.

\begin{tabular}{lcc}
\hline \hline Data set & WMAP code & Modified code \\
\hline$A$ & $0.954 \pm 0.016$ & $0.966 \pm 0.016$ \\
$B$ & $0.958 \pm 0.016$ & $0.969 \pm 0.016$ \\
$C$ & $0.961 \pm 0.014$ & $0.971 \pm 0.014$ \\
\hline \hline
\end{tabular}

other parameters were only subject to small shifts of their mean values.

We repeat this analysis here, but also include data set $\mathrm{C}$ in the analysis. Consistent with Ref. [3] we find that only $n_{s}$ is notably affected by the modified likelihood, and the shift of $n_{s}$ also remains for data set $\mathrm{C}$, in which case the value for $n_{s}$ changes from $n_{s}=0.961 \pm 0.014$ to $n_{s}=0.971 \pm$ 0.014 . This corresponds to a shift of $\sim 0.8 \sigma$, and weakens the significance of $n_{s} \neq 1$ from $\sim 2.9$ to $\sim 2.1$ for this data set. As can be seen from Refs. [2,3], the low- $l$ and pointsource corrections contribute almost equally much to the shift in $n_{s}$. In Ref. [2] they found a mean value of $n_{s}$ of 0.961 when applying only the low- $l$ corrections.

That the shift of $n_{s}$ survives when adding LSS data is not very surprising; $n_{s}$ is less sensitive to LSS than to CMB data because of the larger dynamic range and higher precision of the latter observations. The resulting values of $n_{s}$ are summarized in Table II.

\section{B. Running of spectral index}

Next we consider how the modified WMAP likelihood affects the constraints on $\alpha_{s}$. The simplest inflationary models predict an $\alpha_{s}$ that is slightly different from 0 , and thus information on $\alpha_{s}$ can provide us with valuable information on inflationary mechanisms. Following Spergel et al. [1], we marginalize over the ratio of tensor to scalar fluctuations, $r$, since models with negative $\alpha_{s}$ often correspond to large tensor modes.

Our results are summarized in Table III. We find that the likelihood modifications have no major effect on the constraints on $\alpha_{s}$, but we observe a small increase of $\sim 0.2 \sigma$ in the significance of $\alpha_{s} \neq 0$.

TABLE III. Estimated values for $\alpha_{s}$ from the WMAP likelihood code and our modified code. The other parameters, including $r$, are marginalized over.

\begin{tabular}{lcc}
\hline \hline Data set & WMAP code & Modified code \\
\hline$A$ & $-0.050 \pm 0.027$ & $-0.052 \pm 0.027$ \\
$B$ & $-0.052 \pm 0.026$ & $-0.056 \pm 0.025$ \\
$C$ & $-0.013 \pm 0.020$ & $-0.014 \pm 0.019$ \\
\hline \hline
\end{tabular}




\section{Massive neutrinos}

Another natural extension of the minimal six-parameter model is the addition of massive neutrinos. This is motivated by observations of neutrino oscillations, which show that neutrinos indeed are massive.

Because of their low mass, neutrinos act like a warm dark matter component in the universe. Given the energy fraction of massive neutrinos today, $\Omega_{\nu}$, one can easily find a limit on the sum of the neutrino masses, $M_{\nu}$, by the relation $\Omega_{\nu} h^{2}=M_{\nu} / 93.14 \mathrm{eV}$. (See Ref. [19] or [20] for a review of the cosmological properties of massive neutrinos.)

At present, the best upper limits on the absolute mass scale of neutrinos come from cosmology. The current cosmological 95\% C.L. limits range from $M_{\nu}<0.17 \mathrm{eV}$ [21], relying on extensive use of different data sets, to $M_{\nu} \lesssim 2.0 \mathrm{eV}$ for WMAP data only $[1,22,23]$. In Ref. [22] they also pointed out that it will be difficult to push the upper limit on $\Omega_{\nu} h^{2}$ much below $\Omega_{\nu} h^{2} \lesssim 0.017$ using CMB data only. This corresponds to a neutrino mass limit $M_{\nu} \lesssim 1.5 \mathrm{eV}$. For smaller neutrino masses, neutrinos will still be relativistic at the time of recombination, and thus the effects of the neutrino masses will not be fully revealed in the CMB power spectrum.

In our analysis we assume three species of massive neutrinos with degenerate masses. The assumption of degenerate masses has been shown to be very good for the mass regime that we are working in here [24]. The resulting neutrino mass limits are summarized in Table IV. We see that when using WMAP data alone, the upper limit on $M_{\nu}$ is significantly improved by the modified analysis, and that we are approaching the limit of how tight constraints on $M_{\nu}$ we can find from CMB data alone. If we analyze the data applying only the low- $l$ corrections to the WMAP code, the neutrino mass limit becomes $M_{\nu}<1.69 \mathrm{eV}$, which shows that both the low- $l$ and point-source corrections are important also for this model.

Our improved $M_{\nu}$ limit can be understood by the slight degeneracy between the $M_{\nu}$ and $n_{s}$ parameters, in that a larger value of $n_{s}$ provides less space for a large $M_{\nu}$. This can be seen from the contour plot in the $M_{\nu}-n_{s}$ plane in Fig. 1. The degeneracy can be understood by the fact that both $n_{s}$ and $M_{\nu}$ have impact on the small-scale behavior of the CMB power spectrum.

By definition, $n_{s}$ sets the tilt of the primordial spectrum. If $M_{\nu}$ is of order $\lesssim 2 \mathrm{eV}$, it will affect the power on scales

TABLE IV. Estimated 95\% C.L. upper limits on $M_{\nu}$.

\begin{tabular}{lcc}
\hline \hline Data set & WMAP code & Modified code \\
\hline$A$ & $1.90 \mathrm{eV}$ & $1.57 \mathrm{eV}$ \\
$B$ & $2.13 \mathrm{eV}$ & $1.72 \mathrm{eV}$ \\
$C$ & $0.45 \mathrm{eV}$ & $0.45 \mathrm{eV}$ \\
\hline \hline
\end{tabular}

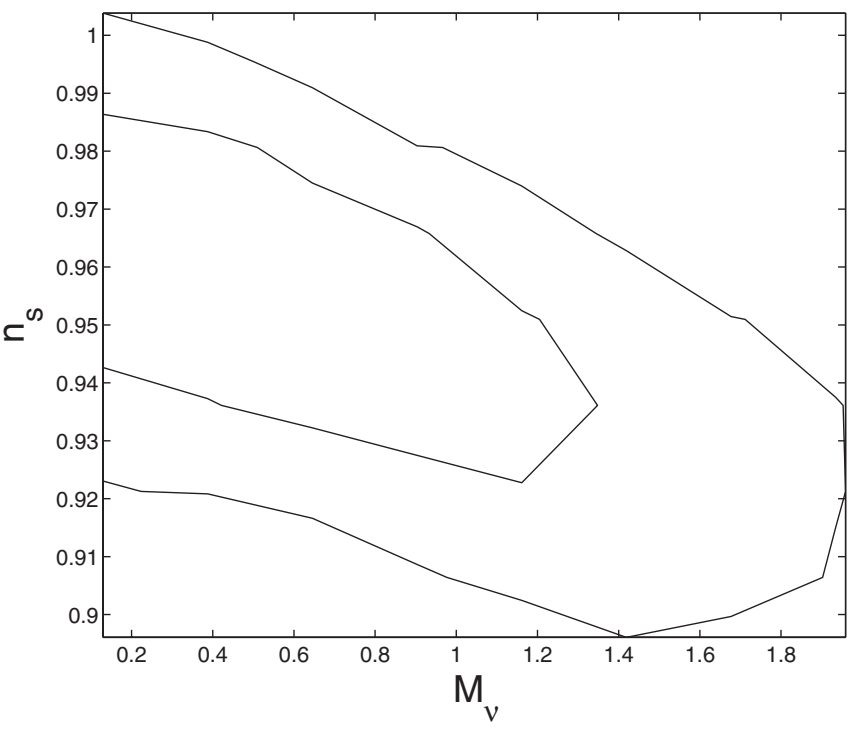

FIG. 1. $68 \%$ and $95 \%$ C.L. contours in the $M_{\nu}-n_{s}$ plane for the modified likelihood code and a seven-parameter model with free neutrino mass, using WMAP data only. There is a slight degeneracy between the two parameters, and larger values for $n_{s}$ will put tighter upper limits on $M_{\nu}$.

smaller than $l \sim 300$. This happens because the perturbations of the gravitational potential on scales smaller than this are suppressed by neutrino free streaming, which in turn boosts the acoustic oscillations [25]. As $M_{\nu}$ increases and more of the dark matter consists of massive neutrinos, this boost of small scale power also increases. Therefore, a large value of $n_{s}$ increases the power on small scales, leaving less room for $M_{\nu}$ to add further power without coming in conflict with data. $M_{\nu}$ also affects the heights of the peaks on larger scales, but this can to a large extent be compensated for by adjusting the values of $\Omega_{m}$ and $\Omega_{b} h^{2}$.

It is interesting to notice that the upper limit on $M_{\nu}$ actually weakens when we add small scale CMB data sets. From Table II we also see that the preferred value of $n_{s}$ also increases when these data sets are included. This indicates that the small-scale power from ACBAR and BOOMERanG is higher than what one would expect from the WMAP data. When adding massive neutrinos to the minimal six-parameter model this increment in smallscale power can be partly accommodated by increasing $M_{\nu}$ instead of $n_{s}$. This will increase the small-scale power without altering the fit to the large scale spectrum.

We also see that when we add LSS and SN1a data, the neutrino mass limit is no longer affected by the modified WMAP analysis, as the additional constraints on $M_{\nu}$ are mainly determined by LSS data. In the LSS power spectrum neutrino free-streaming will suppress small scale power, and a larger $n_{s}$ will in this case allow for a larger $M_{\nu}$. This effect then cancels out the improved $M_{\nu}$ limit found from the WMAP data. 
TABLE V. Estimated values for $\Omega_{k}$.

\begin{tabular}{lcc}
\hline \hline Data Set & WMAP code & Modified code \\
\hline$A$ & $-0.057_{-0.056}^{+0.050}$ & $-0.057_{-0.057}^{+0.050}$ \\
$B$ & $-0.056_{-0.062}^{+0.052}$ & $-0.055_{-0.055}^{+0.048}$ \\
$C$ & $-0.005 \pm 0.007$ & $-0.006 \pm 0.007$ \\
\hline \hline
\end{tabular}

\section{Spatial curvature}

Next we add spatial curvature, $\Omega_{k}$, to our six-parameter model. However, altering the geometry of universe mainly affects the positions of the CMB acoustic peaks, while the likelihood modifications mostly concern the amplitude and tilt of the power spectrum. A priori, one would therefore not expect any significant changes in $\Omega_{k}$. And as seen in Table V, this is indeed the case. For all data sets, $\Omega_{k}=0$ is within $\sim 1 \sigma$, in good agreement with the results from the WMAP team [1].

The large improvement of the limits on $\Omega_{k}$ for data set C can to a large extent be understood by the well-known degeneracy between $\Omega_{k}$ and $h$, where negative values of $\Omega_{k}$ can be accommodated by a small $h$. Therefore, when imposing the HST prior on $h$, the allowed range of $\Omega_{k}$ is significantly constrained.

\section{E. Dark energy equation of state}

The nature of dark energy is one of the major questions in cosmology today. In the minimal six-parameter model, the dark energy is assumed to be a cosmological constant with $w=-1$, and this has been shown to agree well with current cosmological data (see, e.g., Ref. [1]). Here we test whether the modified WMAP likelihood code alters the preferred values for $w$. In the following analysis we assume that $w$ is independent of redshift.

The main effect of $w$ on the CMB power spectrum is to shift the position of the acoustic peaks by altering the expansion history of the universe. Therefore we would not expect the limits on $w$ to be much affected by the new WMAP likelihood analysis. Still, from Table VI we notice a small shift of order $\sim 0.2 \sigma$ to smaller values of $w$ when using $\mathrm{CMB}$ data only. This happens because a smaller $w$ will enhance the late integrated Sachs-Wolfe effect, which results in a suppression of large-scale fluctuations in the observed CMB power spectrum. The slightly smaller preferred value of $w$ in the modified analysis is accompanied by small changes also in $h$ and $\Omega_{m}$ to shift the peaks back in position. As more data is added, we see

TABLE VI. Estimated values for $w$.

\begin{tabular}{lll}
\hline \hline Data set & WMAP code & Modified code \\
\hline$A$ & $-0.98 \pm 0.41$ & $-1.05 \pm 0.39$ \\
$B$ & $-0.97 \pm 0.41$ & $-1.03 \pm 0.37$ \\
$C$ & $-1.00 \pm 0.07$ & $-1.00 \pm 0.07$ \\
\hline \hline
\end{tabular}

that the modified analysis has no effect on $w$ anymore, and that a cosmological constant still fits the data.

\section{F. 11-parameter model}

Finally we vary all 11 parameters $\left\{\Omega_{b} h^{2}, \Omega_{m}\right.$, $\left.\log \left(10^{10} A_{s}\right), h, n_{s}, \tau, \alpha_{s}, r, M_{\nu}, \Omega_{k}, w\right\} \quad$ simultaneously. The results for the parameters $n_{s}, \alpha_{s}, \Omega_{k}, w$ and $M_{\nu}$ are shown in Table VII. Here we see that all effects found in the more restricted models above are also present in this extended model.

For $n_{s}$ the likelihood corrections still result in a mean value that is $\sim 0.02$ larger than with the original likelihood. However, as the uncertainty in $n_{s}$ is increased (mainly due to a degeneracy with $\alpha_{s}$ ), this shift is not as statistically significant as it was in the six-parameter model in subsection III A. Further, for data set $\mathrm{C}$ we see that the preferred value of $n_{s}$ is in fact not much changed by the modified analysis. Rather, the power spectrum changes are accommodated by a slightly lower value of $\alpha_{s}$ to account for the smaller power for low l's in the CMB power spectrum.

For $\alpha_{s}$, there are no significant changes of the preferred values by the 11-parameter model, and we see that $\alpha_{s}=0$ is still consistent with the data.

The same is the case for $\Omega_{k}$. Here the modified analysis shifts the preferred values to slightly more negative values, but with all data sets, $\Omega_{k}=0$ remains well within $1 \sigma$ of its mean value.

Also for $M_{\nu}$ we find the same effects as above. When using CMB data only, the upper limit on $M_{\nu}$ is improved by the new WMAP likelihood analysis. As expected, the limit

TABLE VII. Parameter results for the model that includes free $\alpha_{s}, r, M_{\nu}, w$ and $\Omega_{k}$.

\begin{tabular}{ccc}
\hline \hline Parameter & WMAP code & Modified code \\
\hline \multicolumn{3}{c}{ Data set A } \\
$n_{s}(0.05)$ & $0.863 \pm 0.047$ & $0.880 \pm 0.046$ \\
$\alpha_{s}$ & $-0.051 \pm 0.029$ & $-0.050 \pm 0.029$ \\
$\Omega_{k}$ & $-0.019_{-0.053}^{+0.052}$ & $-0.027_{-0.053}^{+0.049}$ \\
$w$ & $-1.43 \pm 1.09$ & $-1.53 \pm 1.12$ \\
$M_{\nu}$ & $<2.09 \mathrm{eV} @ 95 \%$ C.L. & $<1.66 \mathrm{eV} @ 95 \%$ C.L. \\
& Data set B & \\
$n_{s}(0.05)$ & $0.859 \pm 0.042$ & $0.875 \pm 0.041$ \\
$\alpha_{s}$ & $-0.055 \pm 0.027$ & $-0.055 \pm 0.027$ \\
$\Omega_{k}$ & $-0.010_{-0.050}^{+0.047}$ & $0.018_{-0.053}^{+0.048}$ \\
$w$ & $-1.33 \pm 1.02$ & $-1.43 \pm 1.10$ \\
$M_{\nu}$ & $<2.33 \mathrm{eV} @ 95 \%$ C.L. & $<2.02 \mathrm{eV} @ 95 \%$ C.L. \\
& Data set C & \\
$n_{s}(0.05)$ & $0.954 \pm 0.038$ & $0.954 \pm 0.036$ \\
$\alpha_{s}$ & $-0.003 \pm 0.028$ & $-0.012 \pm 0.026$ \\
$\Omega_{k}$ & $-0.001 \pm 0.012$ & $-0.001_{-0.013}^{+0.011}$ \\
$w$ & $-1.05 \pm 0.09$ & $-1.05 \pm 0.09$ \\
$M_{\nu}$ & $<0.51 \mathrm{eV} @ 95 \%$ C.L. & $<0.52 \mathrm{eV} @ 95 \%$ C.L. \\
\hline \hline
\end{tabular}


becomes weaker for the extended parameter set, but from WMAP data alone we still find a $M_{\nu}$ limit that is better than what is found in earlier papers, even with this large parameter space. Also, we see that the $M_{\nu}$ limit still becomes weaker by adding the ACBAR and BOOMERanG data sets.

For the dark energy equation of state, we still find that the modified analysis prefer slightly lower values for $w$. In this extended model the preferred values of $w$ are shifted to lower values than in the more restricted model in subsection IIIE, which is mainly accommodated by the preferred negative value of $\Omega_{k}$ (as $w$ and $\Omega_{k}$ shifts the positions of the acoustic peaks in opposite directions). Still $w=-1$ remains within $1 \sigma$ for all of the data sets.
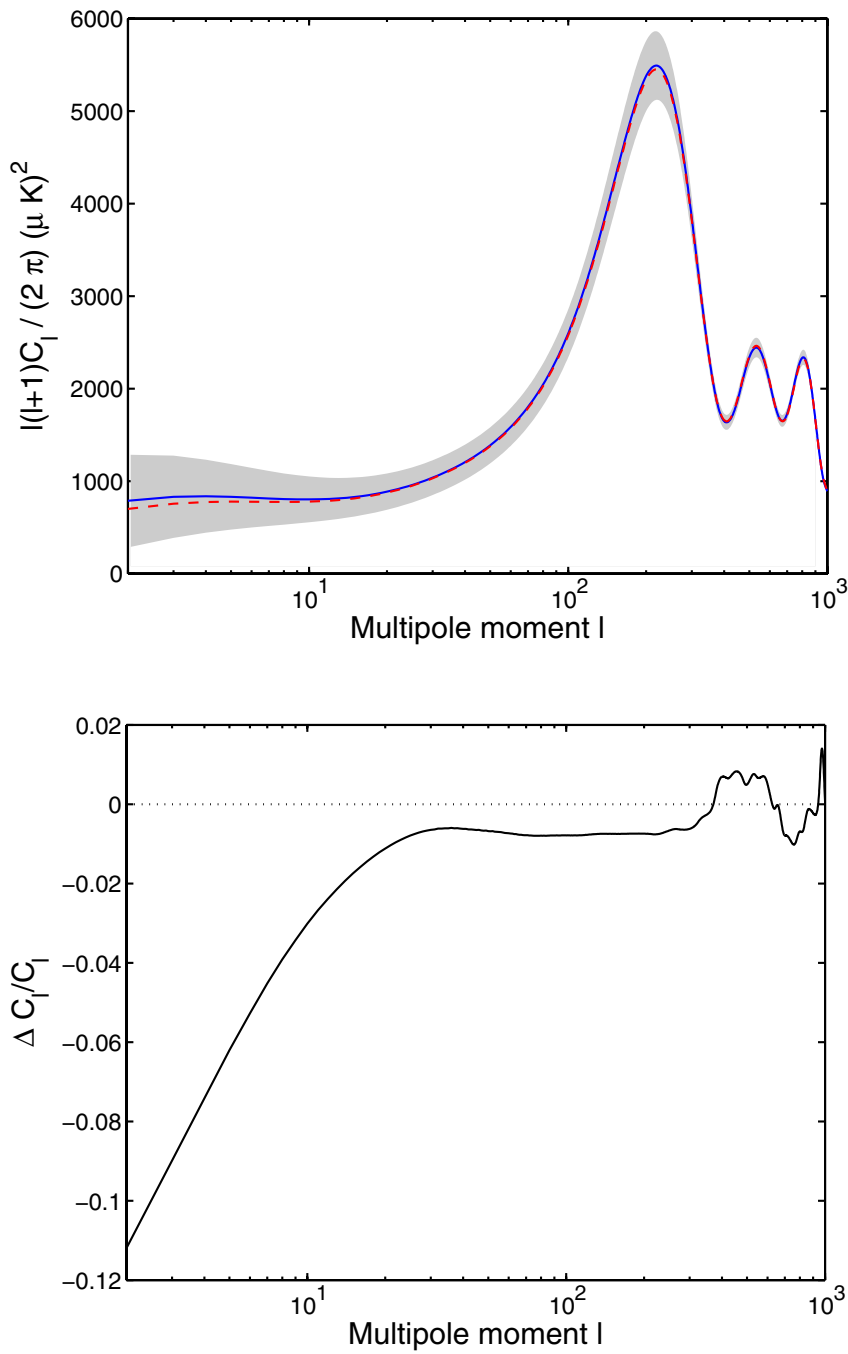

FIG. 2 (color online). Upper panel: The CMB power spectrum for the best-fit 11-parameter model with the WMAP likelihood code (solid blue line) and the low- $l$ and point source corrected likelihood code (dashed red line). The gray shading shows the cosmic variance around the blue line. Note the small discrepancies at both low and high l's. Lower panel: The relative difference between the two power spectra, $\left(C_{l}^{\text {modified }-}\right.$ $\left.C_{l}^{\mathrm{WMAP}}\right) / C_{l}^{\mathrm{WMAP}}$.
In Fig. 2, we show the best-fit power spectrum for the 11-parameter the model both from the analysis with the standard WMAP likelihood code and the new point-source and low- $l$ corrected likelihood analysis. We see that the discrepancy is most notable for $l \lesssim 100$.

\section{CONCLUSION}

In Refs. [2,3], two modifications to the three-year WMAP likelihood were presented. Relative to the power spectrum presented by Hinshaw [4], they found a power deficit for low values of $l$ due to inaccurate likelihood approximation, and a small power excess for high values of $l$ due to over-subtracted unresolved point sources.

The impact on the inferred cosmological parameter intervals from these corrections in a minimal six-parameter model was studied in Ref. [3] using CMB data only. Their single most important result was an increase in the preferred value of $n_{s}$, lowering the significance of $n_{s} \neq-1$ from $\sim 2.7 \sigma$ to $\sim 2.0 \sigma$. In the present paper, we have extended that analysis to also account for cosmological models including a nonzero running of $n_{s}$, massive neutrinos, curvature and $w \neq-1$. We have also added LSS and SNIa data sets to our analysis to see if the parameter shifts induced by the modified WMAP analysis survive when adding more data sets.

We found that the shift of $n_{s}$ to larger values survives when we add LSS and SNIa data. However, when we apply all data sets in the full 11-parameter model, $n_{s}$ is not affected much by the modified WMAP analysis anymore. This is mainly due to the extra freedom with $\alpha_{s}$.

For the extended models, we found that the preferred values of $\alpha_{s}, \Omega_{k}$ and $w$ are not significantly affected by the modified analysis. When including massive neutrinos we found that the upper limit on $M_{\nu}$ when using WMAP data alone was reduced from $M_{\nu}<1.90 \mathrm{eV}$ to $M_{\nu}<1.57 \mathrm{eV}$. A similar improvement in the $M_{\nu}$ limit could not be observed when adding LSS and SN1a data, since the higher preferred value of $n_{s}$ will allow for larger neutrino masses in the LSS power spectrum.

Since the initial publication of the two reanalysis papers, Refs. [2,3], and the present paper, the WMAP team has released a new version of their likelihood code [26] that implements the suggested low- $l$ correction and a revised point-source correction. Using this updated likelihood code, we find $n_{s}=0.959 \pm 0.016$ for the six-parameter model and the WMAP data only. Including massive neutrinos, this code gives an upper bound of $M_{\nu}<1.75 \mathrm{eV}$ from WMAP data only. The difference is due to the pointsource amplitude and corresponding error adopted by the WMAP team, which do not match perfectly that of Ref. [3]. Unfortunately, full details on the WMAP approach are not currently available, and final assessment of this issue must therefore await the release of the revised WMAP3 papers. 
To conclude, the modified analysis does not strengthen the case for nonstandard cosmological parameters, and the standard flat $\Lambda \mathrm{CDM}$ model still provides an excellent fit to data.

\section{ACKNOWLEDGMENTS}

JRK and ØE acknowledge support from the Research Council of Norway through project numbers No. 159637 and 162830. HKE acknowledges financial support from the Research Council of Norway.
[1] D. N. Spergel et al., astro-ph/0603449.

[2] H. K. Eriksen et al., astro-ph/0606088.

[3] K. M. Huffenberger, H. K. Eriksen, and F. K. Hansen, astro-ph/0606538.

[4] G. Hinshaw et al., astro-ph/0603451.

[5] http://lambda.gsfc.nasa.gov; version v2p1.

[6] C.-1. Kuo et al. (ACBAR), Astrophys. J. 600, 32 (2004).

[7] W. C. Jones et al. (BOOMERANG), Astrophys. J. 647, 823 (2006)

[8] F. Piacentini et al. (BOOMERANG), Astrophys. J. 647, 833 (2006).

[9] T. E. Montroy et al. (BOOMERANG), Astrophys. J. 647, 813 (2006)

[10] M. Colless et al., astro-ph/0306581.

[11] M. Tegmark et al. (SDSS), Astrophys. J. 606, 702 (2004).

[12] P. Astier et al., Astron. Astrophys. 447, 31 (2006).

[13] W. L. Freedman et al., Astrophys. J. 553, 47 (2001).

[14] S. Burles, K. M. Nollett, and M. S. Turner, Phys. Rev. D 63, 063512 (2001).
[15] R. H. Cyburt, Phys. Rev. D 70, 023505 (2004).

[16] D. J. Eisenstein et al., Astrophys. J. 633, 560 (2005).

[17] A. Goobar, S. Hannestad, E. Mortsell, and H. Tu, J. Cosmol. Astropart. Phys. 06 (2006) 019.

[18] A. Lewis and S. Bridle, Phys. Rev. D 66, 103511 (2002).

[19] O. Elgaroy and O. Lahav, New J. Phys. 7, 61 (2005).

[20] J. Lesgourgues and S. Pastor, Phys. Rep. 429, 307 (2006).

[21] U. Seljak, A. Slosar, and P. McDonald, J. Cosmol. Astropart. Phys. 10 (2006) 014.

[22] K. Ichikawa, M. Fukugita, and M. Kawasaki, Phys. Rev. D 71, 043001 (2005).

[23] M. Fukugita, K. Ichikawa, M. Kawasaki, and O. Lahav, Phys. Rev. D 74, 027302 (2006).

[24] A. Slosar, Phys. Rev. D 73, 123501 (2006).

[25] S. Dodelson, E. Gates, and A. Stebbins, Astrophys. J. 467, 10 (1996).

[26] Version v2p2p1. 